\title{
Developing TCM-Centered Ecology of Health Care and Maintenance Industries--With the Health Ecosystem around Mt. Qingcheng as an Example
}

\author{
Mujiexin Liu ${ }^{1,2,3}$, Min Liu ${ }^{4,5}$, Dazheng Zhang ${ }^{1,4,5}$ *
}

\author{
${ }^{1}$ Research Center of Qingcheng Medicine, Dujiangyan, Chengdu, 611830, Sichuan, China \\ ${ }^{2}$ Sichuan College of Traditional Chinese Medicine, Mianyang, Sichuan, China \\ ${ }^{3}$ No 5 People's Hospital of Dujiangyan, Dujiangyan, Chengdu, Sichuan, China \\ ${ }^{4}$ Dujiangyan Medical Centre, Chengdu, 611830, Sichuan, China \\ ${ }^{5}$ Chengdu University of Traditional Chinese Medicine, Chengdu, Sichuan, China \\ *Corresponding author. Email: tyu53875251@qq.com
}

\begin{abstract}
By giving a very good example for developing towns into the best destinations for living so as to cope with the fast aging society, this paper aims to illustrate how this can be achieved. Apart from introducing the role of TCM in integrating the limited resources and bringing about new businesses, an $\mathrm{O} 2 \mathrm{O}$ platform that pushes the development of services and industries is also elaborated. The experience can shed light on the construction of such towns in other parts of the country. The model and the platform are new in many ways for building an ecosystem of sustainable development.
\end{abstract}

Keywords: Traditional chinese medicine (TCM), Eco-system, Health care and maintenance, The national

well-being, Development of related industries.

\section{INTRODUCTION}

It is made very clear in the Plan of Promoting Health in China for 2030 as the national strategy to build and develop towns with integrated related industries like health care and maintenance, recreation and tourism into the best ecological destinations for living with health industry at the core. The whole area around Mt. Qingcheng has set up itself an excellent example for developing such towns. Mt. Qingcheng, a renowned AAAAA tourist resort at the altitude of $720 \mathrm{~m}$ with the forest coverage of $59.27 \%$, has the best quality of air containing 22000 negative oxygen ions $/ \mathrm{cm} 3$ and water for health maintenance and postoperative rehabilitation. [1] It is one of the resorts of longevity at the national level with very rich resources of traditional medicine. Apart from these resources, it has a culture and a long history for health maintenance. As the "Garden of Chengdu", back in last century, people in and around Chengdu came here to rent houses for the summer, booming the development of private hostels which in turn attracted even larger amount of people to come to visit, stay for the summer or settle down. The local people who are kind, honest, optimistic and very hardworking makes this place highly attractive to the people from big cities. Even the regular local farm fairs become attractions to the people in Chengdu, who drive here on the weekends to shop for fresh agricultural products, taste local food and visit Mt. Qingcheng. Some people even just come here at the weekends for the good air and quietness. Hence many towns and communities in the area have been fully developed for this market demand, making Mt. Qingcheng a renowned resort of tourism and health maintenance. With the farm-to-table food and vegetables which include many plants both used as food and medicine, people in this area have a long history and rich experience of health maintenance well integrated in their daily life for the recovery and rehabilitation of all kinds of diseases related to chronical health conditions, suboptimal state of health, and postoperative rehabilitation. Based on this history and culture of health care and maintenance, the area of Mt. Qingcheng has been far advanced in integrating the medical treatment and health maintenance, boosting the related industries with TCM as the driving force.

\section{THE ECOSYSTEM OF INDUSTRIES WITH TCM AS THE CENTRE}

Supported by the national policy and the TCM philosophy of "prevention and early intervention at the highest priority", a new type of health care and maintenance is brought about by combining TCM medical treatment and health maintenance. In our fast aging society, with the large and growing senior population, over $75 \%$ are suffering from chronical diseases and those incapable of independent living are exceeding $40 \mathrm{~m}$. Population in suboptimal health conditions is rapidly enlarging that medical resources are too limited to meet the upcoming challenges, making it "very hard to visit doctors and get 
the continued care" even at extremely high costs [2]. The best solution at this moment is to effectively integrate the limited resources so that the efficiency of medical services is optimized. To reach this goal, TCM has its big advantages, especially in the aspect of prevention and early intervention. Being dynamic and individualized, TCM makes it a reality for the full integration of treatment, maintenance and the development of the related services and industries.

Apart from guarding and improving the health and lives for the seniors with health conditions, TCM plays a unique role in the entire population when the whole the nation becomes highly aware of the importance of good health and the high quality of life. Combining the idea and techniques of "prevention and early intervention", people in all age brackets can be guided in the aspects of daily life, good diet habits and positive attitudes towards life [3]. By the principle of TCM, methods of early prevention can be carried out in different groups of people to reduce the occurrence of illness. At the same time, the idea and techniques can be implemented in the whole course of health maintenance for those who suffer from chronical diseases and poor health conditions, and cutting down the number of complications in these aspects. Being natural and low at cost, TCM is the best choice for promoting the health and life quality for the whole population [4].

Propelled by TCM and the local lifestyle, many more hotels and resorts are being built for health maintenance, recreation, breatharianism, meetings and script-writing, from six-star hotels to those only reserved for targeted customers [5]. All of them provide uniquely designed cuisine based on TCM philosophy to attract more customers. People have a wide variety of activities to choose form in the area, such as visiting the National Park of Panda for fun and research, the small towns for food, tea and experiencing the local life. To meet the high demand of the tourism in this area, the first inter-city rails was built in 2008 [6]. M-TR is being built at the moment for the growing market [7]. Because of the wonderful environment, many large institutions and enterprises have come and settled here [8,9]. And international events like WCH \&WNH (World Cultural Heritage and World Natural Heritage) Marathon are held here each year, making this area a world-renowned resort of health maintenance [10]. In recent years, many people, both from home and abroad, come here on purpose for TCM treatment because No.5 People's Hospital of Dujiangyan is very famous for treating allergies and ocular diseases with TCM.

The TCM ecological circle around Mt. Qingcheng, has already formed into a service system that promotes all the related industries. Applying the principle that medicine and food are of the same roots, this system pushes forward the demand for and rely on the traditional medicines, which largely boosts the production and processing of the traditional medicines [11]. With TCM health maintenance as the leading role, the related industries of tourism, culture, agriculture and forestry are all brought together to meet the different needs of the market, creating new forces for economic growth. In the Demonstration Area of TCM Service and Health Maintenance for Tourism in Dujiangyan with Mt. Qingcheng as the major part, there have been many theme-hotels, theme-parks, famous streets, towns and resorts providing services and products of traditional medicine, food for medical purposes and health maintenance [12]. (See Figure 1)

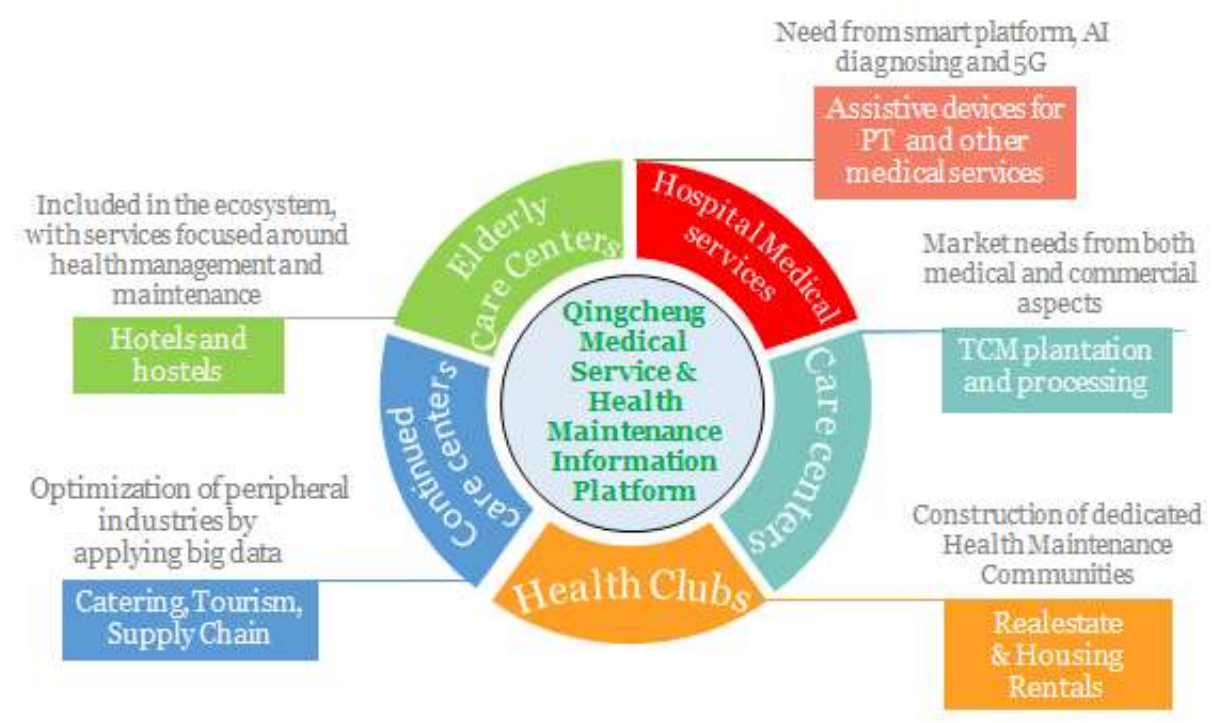

\section{Qingcheng Health Maintenance Ecosystem}

Figure 1 Illustration of Qingcheng health maintenance ecosystem 


\section{TCM-CENTERED PLATFORM DRIVES THE COORDINATE DEVELOPMENTS AMONG INDUSTRIES}

With the rising living standard and people's awareness of health, Qingcheng ecological circle of health care and maintenance does not only push forward the coordinate development of the medical institutions with increasing efficiency but also integrate all the resources in the surrounding areas to form a system centered on TCM and other medical treatment and services in many dimensions [13]. On the basis of the local rich resources and related industries, all the information of the private and star hotels, real estates are built into a database for analysis, selection to enable all institutions and business to provide the needed services and products for the travelers and local residents. Supported by the service platform and big data, the portraits of users are generated, which further enhances the coordination of services online and offline so that more hostels and businesses are connected into a full-ledged ecosystem to meet the demands with services improved upon feedbacks [14]. Naturally, this area is not just a place for people to stay for summer, but a most agreeable habitat for people to settle down or stay longer for comprehensive recovery, especially after operations. In recent years, the housing sales and rentals are soaring in this area of well-developed communities. So are those of the medical devices and continued care services. Thus, the O2O-based platform of big data gives rise to all the related business in and around this area [15]. The unique characteristics of TCM prevention and early intervention is exponentially amplified in the ways of taking accurate, dynamic and active measures to match the demands of all age groups for medical services. Together with a well-developed customer service system, user needs, ratings and user favorability are better understood for future development. The upcoming 5G technology allows the virtual and physical experience for the customers to get the most suitable services and packages. Even for the elderly, VR allows them to have a global view of institutions and their services. Their needs can be tracked and followed for better and more accurate services offline [16]. This model is timesaving and effective for both customers and the businesses, especially when the whole society is taken into consideration. People of different age brackets at offices, homes, centers of continued care, recreation, health maintenance and people in the resort can get the information and services both online and offline.

\section{TCM LEADS THE DEVELOPMENT OF SERVICES}

Trying to establish a $15 \mathrm{~m}$-service-circle, this platform at its center fully integrates the medical treatment and heath maintenance, the resources of the elderly care, continued care and medical service for the communities so that it becomes possible to provide the individualized and customized services for those who need post-operative rehabilitation, post-operative TCM recuperation, recovery, or continued care without having to leave their own familiar living environment [17]. Attracting more people to come for these services, this $15 \mathrm{~m}$-service-circle can bring about the development of multiple services and push the related industries to become more professionalized and complete through competition for the quality of services provided.

This model of Internet + medical treatment and health maintenance promotes the industries and their services. With concert efforts, they can bring about the development and the construction of healthy cities with highly integrated service structures. To support these developments, many more production bases of traditional Chinese medicine, more resorts for medical treatment and health maintenance, and service centers are being built to fulfil the market demands. Eventually, the development of the services will push the ecosystem to become larger and better with clusters of good enterprises and industries. Based on the development of the market demand, the medical research on TCM and its derived products will be further improved, which in turn will create and accelerate the development and promotion of new services concerning green food, better medical products and devices, and even supply chain and logistics development. The development of related services will for sure make this area more attractive to people at home and abroad as a resort of health and tourism. Hotels, travel agencies, and places of interest will all benefit from the development and become the most active agents in this TCM centered ecological system.

\section{CONCLUSION}

After the pandemic of COVID-19, the demand for health care and maintenance will be further upgraded with the increasing awareness among the whole population about good health and getting fit. It will be an indisputable investment with great potentials in the sectors of effective allocation of health care resources, better and more complete system for medical care services and more comprehensive systems for management. More importantly, TCM will push forward the sustainable development of the ecosystem with all the related industries of tourism, catering, transportation, logistics, renting business and the real estates.

\section{ACKNOWLEDGEMENT}

This paper was supported by The Youth Scientific Innovation Foundation of Sichuan Chinese Medicine Administration (2016Q080). 


\section{REFERENCES}

[1] Building the First Tourism Resort of Health Care and Maintenance in Dujiangyan [EB/OL] https://epaper.scdaily.cn/shtml/scrb/20150525/100288.s html.

[2] The average longevity at 68.7 and the population above 60 over $250 \mathrm{~m}$ [EB/OL].

https://health.huanqiu.com/article/7RgF3cYSvUA,2019 $-11-1$.

[3] W J Liu, X W Sun, L Zhang. The Necessity of Constructing the Model of "Combing Health Care and Maintenance" That Covers the Whole Population[J]. Chinese Health Economics, 2016,35(01):35-37.

[4] L Lin, W B He. Discussing Application of Yin and Yang Five Elements Theory in Preventive Treatment of Disease[J]. Journal of Liaoning University of Traditional Chinese Medicine, 2013, 015(006):134-135.

[5] X F Liu. Research on tourism real estate of Dujiangyan[D]. 2015.

[6] S Yang, C Y Zhang. The Study on the Influence on the Tourism Development Brought about by the Dujiangyan-MTR[J]. Tourism Overview, 2015(07):165-166.

[7] The Commencement of Dujiangyan M-TR that Links Mt. Qingcheng and the Panda Valley [EB/OL] https://baijiahao.baidu.com/s?id=159865118070163018 $6 \& w f r=$ spider $\&$ for $=$ pc.

[8] Dujiangyan City Government Work Report 2019-02-02 [EB/OL]

http://www.djy.gov.cn/dyjgb_rmzfwz/c129765/2019-05 130/content_559cd12cc69945dfa0a9249e75288ff9.shtm 1 .

[9] A Report on Boosting the International Garden City for Health Maintenance [EB/OL] https://new.qq.com/omn/20190227/20190227B0A91Z.h tml.

[10] C Xie, X L Li, S Liu. An Empirical Study on the Impact of Sports Events on Tourism[J]. Journal of Nanjing Institute of Physical Education and Sports, 2017, 016(001):139-143.

[11] M Tang, C Y Zhang, H B Wu. A Comparative Study on the Model of Combining Medical Treatment and Health Maintenance[J]. Chinese Health Economics, 2018(2):60-63.
[12] Q Li. Ideas and Suggestions on Building the Town Mt. Qingcheng into Health Maintenance Resort[J]. Business Economy, 2018, No.504(08):51-54.

[13] M Tang, H B Wu. A Study on the Model Based on the Combination of Medical Treatment and Health Maintenance[J]. Chinese Health Economics, 2017(5):22-24.

[14] H L Zhu. The Ideas and Innovation on the Continued Care of the Elderly in China [J]. Journal of Social Science of Hunan Normal University, 2016,45(3):68-73.

[15] W Dai, X Y Zhang, X W Sun. The Model of "Combining the Medical Treatment and Health Care" from the Perspective of the Well-being of the Whole Population in China[J]. China Social Security, 2015(10):32-32.

[16] Sukhmani S, Sadeghi M, Erol-Kantarci M, et al. Edge Caching and Computing in 5G for Mobile AR/VR and Tactile Internet[J]. IEEE Multimedia, 2019, 26(1):21-30.

[17] F Guan, Z X Sun, J Lin, et.al. A Preliminary Study on the Construction of Smart Medical Care[J]. Modern Hospital Management, 2013,11(2):28-29. 